and only 7 had been obliged to seek lighter employment; of 7 operated patients only 2 are fully capable of working. In the reference to Dr. Boysen's review ${ }^{2}$ some significant figures have been omitted : $86.5 \%$ (225 out of 260 ) have complete working capacity, while 30 are fit for light work. Only 5 are unable to work. These figures agree with those of Kuhns," who found that 790 out of 1,000 patients recovered completely from sciatica without operation.

If, as Prof. Jefferson emphasizes, the clinical picture of disk protrusion does not differ at all from that of the cases in which no protrusion was found at operation, it is possible that the pain is not due to the protrusion. This possibility is strengthened by the recent demonstration that complete recovery is the rule after a negative exploration of the lumbosacral canal. ${ }^{45}$

Most of the optimistic reports of operation on intervertebral disks are based on surveys conducted by questionary. We should welcome, therefore, the recent appearance of two reports based upon the physical examination of the patients by practitioners who had not performed the operation : (i) Aitken and Bradford $^{6}$ : results good in 50 out of 170 cases; $45 \%$ unable to return to work. (ii) Lenhard ${ }^{7}$ (reviewing the work of the late W. E. Dandy) : only 35 out of 147 are entirely well.-I am, etc.,

Melbourne.

Michael Kelly

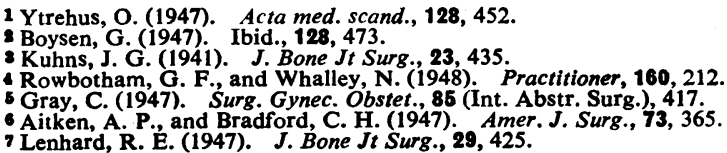

\section{Termination of Pregnancy}

SIR,-Women desirous of having their pregnancies terminated on psychiatric grounds generally fall into one or other of the following groups : $(a)$ the frankly neurotic ; $(b)$ those who have previously had a psychotic illness; $(c)$ the pre-menopausal, often associated with $(a)$ or $(b) ;(d)$ the unmarried; $(e)$ the married, with as many children as they can reasonably manage (inadequate housing, economic factors, etc., constitute very real problems in this group); $(f)$ those who attempt to evade their normal and natural responsibilities on some frivolous pretext. The syndromes presented by each group are fairly constant for that group. Each case should be decided on its merits, and it is important to obtain as much corroborative evidence as possible before reaching a decision, which, as Dr. Chesser so aptly puts it (June 5, p. 1110), is " practically always a choice between a greater and a lesser evil."

Childbirth is the natural function of women, and any reluctance on a woman's part to carry on with a pregnancy must be viewed with concern. We should not think merely in terms of a condition reactive to an unwanted pregnancy. If we do so we may fail to identify a deep-seated disorder, which may involve the patient in a mental breakdown whether her pregnancy is terminated or not. The most difficult cases to assess are the unmarried women with stable past histories. The social and economic consequences of having an illegitimate child obviously have more violent repercussions upon them, by and large, than does the advent of an unwanted child to a married woman. The unmarried unwillingly pregnant woman frequently commits suicide, while her married contemporary rarely does so.

As previously mentioned, the acute disturbance precipitated by an unwanted pregnancy may readily obscure a serious disorder of personality. The relief afforded by a therapeutic abortion tends to produce a false sense of security in such cases. Before reaching a decision, therefore, on the desirability of terminating a pregnancy, a comprehensive psychiatric investigation is called for, preferably spread over a number of interviews. It has been difficult in the past to insist on further treatment after the surgical interference has been concluded. but with the advent of the National Health Service psychiatric treatment should be available to patients who formerly could ill afford it. Psychological treatment is as important after therapeutic abortion on psychiatric grounds as is the re-education and rehabilitation of a patient after amputation.-I am, etc.

London, W.1.

Ellis Stungo.

\section{Stiff Test for Nurses}

SiR,--In connexion with the current discussion on the training of nurses I should like to draw attention to certain questions in the fevers paper recently set for the Final State Examination of the General Nursing Council.

1. "Which infectious diseases may be complicated by involvement of the central nervous system ; what are the special risks and how may the diagnosis be confirmed ?"

2. "Describe the appearance, cultural characteristics, and properties of the diphtheria bacillus."

Apart from the scope, ambiguity, and complexity of the first question, there arises an important point of definition. In regard to the second question an adequate answer would involve a knowledge of bacteriology quite beyond that which should be required from student nurses. In fact, the Council's syllabus merely refers to the "causation" of diphtheria, and a standard textbook in popular use among nurses deals with the subject in a few lines.

Questions such as these produce a sense of frustration and not unreasonable indignation among candidates. As one responsible for the training and recruitment of nurses I suggest that the requirement of such academic standards of medical and bacteriological knowledge does not tend to produce or discover good practical nurses, nor does it help to overcome existing difficulties in regard to nursing shortage and lack of recruits to the profession.-I am, etc.,

Ilford.

J. H. WEIR.

\section{Convalescent Homes}

SIR,-We see all round us the deplorable results of sectionalism, both at home and in the international field. In a small way we may combat this evil by taking care that it is not made part of medical planning. I have been struck by the opportunities presented when patients are sent away to convalesce.

Recovery is inevitably assisted by stimulating surroundings and contacts. It is therefore regrettable that in so many places convalescents are collected from one small occupational group, whereas if persons in a variety of different employments were brought together they would, from the very diversity of their interests, stimulate one another while broadening their minds. In this way some little may also be done to remove those distrusts and animosities which derive from a narrow and restricted way of life. For these reasons it appears desirable to make the most of an opportunity to secure on the same occasion a more rapid physical and mental recovery with, as one may hope, an increase in social and political sense. -I am, etc.

Hadley Woods, Herts.

G. C. Pether.

\section{Short Leg in Soldiers}

SiR,-We read with interest Dr. Nesta $H$. Wells's letter (June 19, p. 1206) on scoliosis in school-children and her plea for early compensation for leg shortening by heel raising. In dealing with recruits in the Army who are sent to the Army Physical Development Centre we have found a high incidence of leg shortening with resultant scoliosis. The recruits sent here are mostly in the $17 \frac{1}{2}-18 \frac{1}{2}$-years age group. The shortening varies from $1 / 4$ in. to 11 in. $(0.63 \mathrm{~cm}$. to $3.17 \mathrm{~cm}$.). Remarkably few give any history of backache, sciatica, or bony injury to the lower limbs, or of poliomyelitis. Even fewer are aware prior to our examination that there is any shortening.

It is our practice, however, whether symptoms are present or not, to compensate for shortening by heel raising as a prophylactic measure against back pain, except where the shortening is $1 / 4 \mathrm{in}$. or less. Such shortening we regard as a normal variation. In all cases where symptoms are present, and especially in youths of this age group, it is most important that other causes of low back pain, including ankylosing spondylitis. are ruled out. We therefore make it a rule in these cases to have an $x$-ray examination of the spine and sacro-iliac joints.

Shortening of between $1 / 2 \mathrm{in}$. and $1 / 4 \mathrm{in}$. we treat by raising the heel on the affected side by that amount. Heel raising of more than $1 / 2$ in. gives rise to a somewhat cumbersome boot. Therefore. when the shortening is of more than $1 / 2$ in., we find 